\title{
PERSPECTIVA DE ADOLESCENTES QUE VIVEM COM DIABETES MELLITUS ACERCA DO AUTOCUIDADO
}

\author{
Caren da Silva Bertoldo', Eliane Tatsch Neves', Aline Cammarano Ribeiro1, Camila Barreto1, \\ Diúlia Calegari de Oliveira' ${ }^{1}$, Camila Lopes Marafiga ${ }^{2}$ \\ 1Universidade Federal de Santa Maria, Brasil. carensbertoldo@gmail.com; eliane.neves@ufsm.com; \\ alinecammarano@gmail.com; camilabarreto_6@msn.com, diuliacoliveira@gmail.com \\ ${ }^{2}$ Faculdade Integrada de Santa Maria, Brasil. Camilal_marafiga@outlook.com
}

\begin{abstract}
Resumo. Introdução: a Diabetes Mellitus tipo 1, caracteriza-se como uma doença autoimune com expressivo quantitativo de crianças e adolescentes acometidos. A adolescência é permeada por inúmeras descobertas, junto a isso, os adolescentes com diabetes mellitus tipo 1 precisam adaptar-se ao cotidiano de convívio com a rotina de cuidados imposta pela doença crônica. Com isso, torna-se pertinente o conhecimento sobre como o adolescente desenvolve o autocuidado. Objetivos: conhecer como os adolescentes que vivem com diabetes mellitus tipo 1 desenvolvem 0 autocuidado. Métodos: este estudo configura-se como pesquisa qualitativa, exploratória e descritiva. A coleta de dados ocorreu em um hospital de ensino da região sul do Brasil, no ambulatório de pediatria. Os participantes foram adolescentes com diabetes mellitus tipo 1, de idade entre 12 anos e 18 anos incompletos. Realizou-se 14 entrevistas. As entrevistas foram transcritas e submetidas à análise temática de conteúdo, segundo Braun e Clarke (2006). Resultados: A partir da análise emergiram os seguintes temas: "Percepção de autocuidado"; "Alterações nos hábitos de vida" e "Prática de exercícios físicos", sendo agrupados na categoria temática: "Autocuidado de adolescentes que vivem com Diabetes Mellitus". Conclusões: os adolescentes percebem o autocuidado como as atividades diárias que eles realizam e possuem autonomia na sua realização.
\end{abstract}

Palavras-chave: Autocuidado; Doença Crônica; Diabetes Mellitus; Adolescentes; Pesquisa Qualitativa.

\section{PERSPECTIVE OF ADOLESCENTS LIVING WITH DIABETES MELLITUS ABOUT SELF CARE}

\begin{abstract}
Background: Type 1 Diabetes Mellitus, is characterized as an autoimmune disease with significant numbers of affected children and adolescents. Adolescence is permeated by innumerable discoveries, along with this, adolescents with type 1 diabetes mellitus need to adapt to the daily life of living with the care routine imposed by chronic disease. With this, knowledge about how adolescents develop self-care becomes relevant. Objective: to know how adolescents living with type 1 diabetes mellitus develop self-care. Methods: this study is a qualitative, exploratory and descriptive research. Data collection took place in a teaching hospital in the southern region of Brazil, at the pediatric outpatient clinic. Participants were adolescents with type 1 diabetes mellitus, aged between 12 and 18 years old. There were 14 interviews. The interviews were transcribed and submitted to thematic content analysis, according to Braun and Clarke (2006). Results: From the analysis, the following themes emerged: "Perception of self-care"; "Changes in lifestyle habits" and "Physical exercise practice", being grouped in the thematic category: "Self-care of adolescents living with Diabetes Mellitus". Conclusions: adolescents perceive self-care as the daily activities they perform and have autonomy in carrying it out.
\end{abstract}

Keywords: Self Care; Chronic Disease; Diabetes Mellitus; Adolescent; Qualitative Research.

\section{INTRODUÇÃO}

A DM1 é definida como doença autoimune, acontece quando a produção de insulina do pâncreas é insuficiente, pois suas células sofrem destruição. Os portadores de DM1 
apresentam demandas de cuidados diários, necessitam de injeções de insulina para manter a glicose no sangue em valores normais, teste da glicemia capilar e controle da alimentação (Bertin, Elizio, Moraes, Medeiros, \& Soo 2016). É uma doença que resulta em demandas clínicas, psicológicas e sociais na vida das pessoas. A doença se caracteriza por poliúria, polidipsia e emagrecimento apresentados por grande parte dos indivíduos acometidos. A DM tipo I é mais comum se manifestar na infância e na adolescência. O público infantojuvenil acometido pela doença, vivência diversos percausos em relação à faixa etária e suas atribuições sociais (Bertonhi \& Dias, 2018). Na adolescência as necessidades da doença somam-se as características da fase. O adolescente vivencia mudanças físicas, psicológicas, sociais e comportamentais com a necessidade de integrações sociais e descobertas pessoais e ao mesmo tempo precisa atentar-se para os cuidados com a sua saúde para evitar o adoecimento.

Dessa forma o adolescente acometido pela DM1 vivencia diversas mudanças no seu cotidiano, as quais interferem no seu processo de desenvolvimento, tais como a dieta restrita, aplicações de insulina diariamente, atividade física regular, sentimento de medo das possíveis complicações e mal-estar pelos sintomas de hiperglicemia e hipoglicemia. Nesse contexto, o adolescente se vê obrigado a amadurecer precocemente, pois depende de ações de autocuidado para manutenção do processo saúde-doença que tem como agravante as características inerentes da idade. A partir de tal conjuntura, evidencia-se a importância do autocuidado na rotina desses adolescentes que possuem um conjunto de regras pré-estabelecidas, a fim de promover a própria saúde e bem-estar (Andrade, 2014).

Nessa perspectiva, é imprescindível a atuação dos profissionais da saúde no cuidado ao adolescente que vive com DM1, principalmente do profissional enfermeiro, visto que atua diretamente na rotina dos usuários e seus familiares de modo a conhecer suas reais necessidades de cuidado.

Assim, torna-se necessário o trabalho conjunto entre profissionais e adolescentes, a fim de promover o acolhimento, diminuir a ansiedade e promover o empoderamento para 0 autocuidado assistido.

A partir do exposto tem-se o questionamento: como os adolescentes que vivem com diabetes mellitus tipo 1 desenvolvem o autocuidado? Assim, objetiva-se: conhecer como os adolescentes que vivem com diabetes mellitus tipo 1 desenvolvem o autocuidado. 


\section{METODOLOGIA}

Este estudo configura-se como pesquisa qualitativa, exploratória e descritiva. A pesquisa qualitativa objetiva a compreensão dos fenômenos que se relacionam aos seres humanos, assim utiliza-se de valores e percepções na busca pelo conhecimento, ou seja, ela analisa expressões humanas intrínsecas em suas relações. Ademais, a pesquisa qualitativa preocupa-se em garantir a cientificidade das produções por meio de quatro critérios: coerência, consistência, originalidade e objetivação, os quais devem ser observados e respeitados ao se pesquisar (Minayo, 2014).

Realizada no ambulatório de um hospital localizado em município que possui cerca de 280.000 habitantes. Trata-se de um hospital de ensino vinculado a uma Universidade Federal, credenciado ao SUS, referência no atendimento em saúde para toda a macrorregião centro-oeste do estado do Rio Grande do Sul. O ambulatório recebe uma grande demanda de adolescentes para realizarem tratamento em saúde em diversas clínicas e especialidades, como, seguimento de prematuro; Cirurgia pediátrica; Doenças infecciosas pediátricas; Diabetes infantil; Endocrinologia pediátrica; Grupo de obesidade; Neurologia estimulação precoce; Neurologia pediátrica; Obesidade criança e adolescente; PAISC (Programa de Assistência Integral à Saúde da Criança); Pediatria; Pneumologia pediátrica.

O serviço de saúde funciona de segunda a sexta-feira, nos horários das $7 \mathrm{~h}$ às $19 \mathrm{~h}$ e os usuários são encaminhados pelo sistema de referência e contra-referência da Secretaria Municipal de Saúde dos municípios com o hospital universitário. Conta com uma ampla equipe multiprofissional e uma área física constituída por nove consultórios, uma sala de enfermagem, uma recepção, uma sala de estar para equipe de enfermagem e uma brinquedoteca.

Os participantes desse estudo foram adolescentes com diabetes mellitus tipo 1, de idade entre 12 anos e 18 anos incompletos (Brasil, 2015), que realizaram pelo menos uma consulta no ambulatório durante o período de coleta de dados. Assim, realizou-se 14 entrevistas obedecendo aos critérios de inclusão. Ademais, três adolescentes se recusaram a participar do estudo e um teve consentimento do responsável negado. A coleta desses dados aconteceu no primeiro semestre de 2017 por meio da entrevista com roteiro aberto, composta pela seguinte questão norteadora: Conte-me como você desenvolve seu cuidado em relação à diabetes mellitus no dia-a-dia. Essa técnica de obtenção de dados tem como 
finalidade dar voz aos participantes do estudo sobre a maneira como desenvolvem o autocuidado no seu cotidiano.

As entrevistas, como meio de obtenção de dados qualitativos, permitem captar informações pessoais detalhadas e proporcionam informações permeadas pelos pontos de vista dos participantes (Creswell, 2013). A opção pela entrevista aberta, sem categorias preestabelecidas, foi definida para que os participantes expressem da melhor maneira suas experiências, sem serem influenciados pela visão do pesquisador ou pelos resultados de outros estudos, além de proporcionar flexibilidade para o pesquisador trabalhar com as questões de acordo com o ritmo e conteúdo oferecidos pelo participante durante seu discurso.

Os adolescentes foram abordados no ambulatório do hospital, cenário do estudo, durante o período de espera pelas consultas de rotina previamente agendadas com o médico endocrinologista. A primeira abordagem foi realizada na sala de espera e posteriormente os participantes eram direcionados para uma sala reservada. Alguns pais acompanharam os filhos durante as entrevistas, ao passo que outros não julgaram necessário, mesmo assim, todos os responsáveis assinaram o Termo de Consentimento Livre e Esclarecido (TCLE), consentindo a participação do adolescente na pesquisa, e o adolescente assinou o termo de assentimento, concordando em participar. A coleta de dados foi encerrada quando a pesquisa alcançou a densidade teórica de acordo com Moreira e Caleffe (2008) para atender os objetivos do estudo.

As entrevistas duraram entre 15 minutos até 30 minutos, foram gravadas em áudio, transcritas na íntegra pelas pesquisadoras, identificadas por letras e números (identificação alfanumérica) e, posteriormente, submetidas à análise temática de conteúdo, segundo Braun e Clarke (2006), a qual se divide em seis etapas. Familiaridade com os dados: transcrição das entrevistas e releituras dos dados; Geração de códigos: codificação de forma sistemática (agrupamento) das características dos dados em comum, sendo os códigos gerados: "autocuidado"; "hábitos de vida" e "exercícios físicos". Procura de temas: formação de temas potenciais; Revisão de temas: verificação da relação dos temas com os dados (nível 1) e geração da temática para análise (nível 2); Definição e Nomenclatura dos temas: análise para refinar a especificidade de cada tema, geração e definição de nomes para cada tema; Elaboração do relatório: sendo esta a última oportunidade para análise dos extratos. Trás a análise para a pergunta da pesquisa e permite a redação final de um 
relatório científico. Os autores destacam que não é necessário seguir a ordem descrita, o pesquisador tem a liberdade de retornar etapas sempre que necessário (Braun \& Clarke, 2006). Cabe destacar que a codificação dos dados foi realizada pela pesquisadora principal para imersão necessária dos dados.

Foram preservados os direitos dos informantes observando-se as orientações da Resolução no 466/12 do Conselho Nacional de Saúde, que trata das Diretrizes e Normas Regulamentadoras de Pesquisa Envolvendo Seres Humanos (Brasil, 2012). A pesquisa passou por avaliação do Conselho de Ética e Pesquisa da Universidade Federal de Santa Maria (CEP/UFSM) e recebeu aprovação sob o parecer $\mathrm{n}^{\circ}$ 2.233.073, no CAAE: 57774916.7 .0000 .5346 .

\section{RESULTADOS E DISCUSSÃO}

\subsection{Autocuidado de adolescentes que vivem com diabetes mellitus}

Participaram do estudo 14 adolescentes, dez do sexo masculino e quatro do sexo feminino. A idade média dos participantes foi de 14 anos. Todos possuem dignóstico de DM tipo 1 e fazem acompanhamento frequente no ambulatório do hospital cenário do estudo. A maioria deles faz uso de insulinoterapia para manutenção adequada da glicemia. A partir da análise temática indutiva emergiram os seguintes temas: "Percepção de autocuidado"; "Alterações nos hábitos de vida" e "Prática de exercícios físicos". Os temas foram agrupados na categoria temática: "Autocuidado de adolescentes que vivem com Diabetes Mellitus".

Os adolescentes entrevistados percebem o autocuidado como as atividades diárias que eles realizam em detrimento das mudanças de hábitos ocasionadas pela Diabetes Mellitus.

"Eu levanto, me escovo, como as coisas que tenho que comer, faço insulina, faço as coisas dentro de casa." (A2, 13 anos)

"[...] É, eu acordo, tem que fazer o HGT (Hemoglicoteste) e aplicar a insulina e uma tal de regular. Depois eu tomo café, foi a coisa que mais mudou, por causa que eu tive que tirar uns tipos de pão e comer só integral, é a pior coisa que tem. Depois, chega o meio dia e eu tenho que fazer de novo o HGT (Hemoglicoteste). Depois do meio dia tem outro lanche da tarde, que eu tenho que fazer se não fica muito baixa (insulina). Chega a janta e eu tenho que fazer o mesmo procedimento que é HGT (Hemoglicoteste) e a regular $[\ldots] "$ " $(A 3,13$ anos) 
Para muitos adolescentes o processo de adolescer é complexo e conflituoso, uma vez que ocorrem diversas alterações físicas, biológicas, sociais e psicológicas. Para os adolescentes que vivem com diabetes mellitus esse processo é dificultado pelo diagnóstico de uma condição crônica, que quase sempre é motivo para ansiedade, preocupação, tristeza e insegurança por parte dos adolescentes (Ferreira, Zanatta, Brum, Nothaft, \& Soo, 2013; Conte \& Jeneral, 2018). Esses sintomas emocionais são atribuídos às alterações que a diabetes mellitus implica no cotidiano dos adolescentes, principalmente no que diz respeito a rotina de autocuidado relacionada aos hábitos alimentares, a prática de exercícios físicos, a monitorização da glicemia e a aplicação da insulina, restringindo alguns hábitos aos quais já estavam em sua rotina (Greco-Soares \& Dell'Aglio, 2016; Greco-Soares \& Dell'Aglio, 2017).

Desse modo, a autoaplicação da insulina e a realização do controle glicêmico através do HGT (Hemoglicoteste) esteve presente no discurso dos adolescentes ao se reportaram acerca da maneira como desenvolvem seu autocuidado.

"Quando a insulina entrou (começou a usar), eu aplico uma vez por dia de manhã." (A6, 16 anos)

"Os HGTs (Hemoglicoteste) eu faço, ah.. quando é pra aplicar na perna, na barriga, isso ainda eu aplico. Quando é pra aplicar no braço eu não consigo, dai alguém aplica. Na escola eu só meço HGT, em casa eu faço controle com insulina e HGT e eu continuo fazendo uma contagem de carboidratos." (A5, 14 anos)

"A insulina eu faço a basal de manhã, a Lantus, e ai faço a de apoio, a ultrarrápida, ai depende o que eu vou comer durante o dia, mas no mínimo umas cinco vezes no dia. Os HGT (Hemoglicoteste) é conforme eu vou fazer a refeição ou quando eu sinto se ela tá alta ou tá baixa." (A4, 17 anos)

Com relação a aplicação da insulina, seja autoaplicada ou não, e o monitoramento da glicemia capilar com o hemoglicoteste (HGT), as autoras Greco-Soares e Dell'Aglio (2017) acreditam que esses são fatores que podem influenciar negativamente na adesão ao tratamento e a rotina de autocuidado do adolescente que vivem com diabetes mellitus, visto que, são procedimentos que implicam dor e desconforto ao usuário. Isso o desmotiva e pode culminar em sintomas psicológicos a longo prazo, como por exemplo a depressão. Apesar disso, a literatura aponta a importância da independência do adolescente na realização dos cuidados relacionados à $\mathrm{DM}$, uma vez que a autonomia caracteriza-se como a capacidade do indivíduo de gerenciar sua própria vida, de modo a torná-lo capaz de decidir por si próprio e ser responsável por sua saúde. Além disso, a autonomia também constitui-se como importante aliado na adesão ao tratamento e realização do autocuidado impactando de maneira positiva na qualidade de vida dos adolescentes (Espírito et al., 2012; Greco-Soares \& Dell'Aglio, 2016). 
A partir disso, observam-se dois fatores importantes para o manejo da DM, a adesão ao tratamento e o autocuidado que embora estejam correlacionados, possuem sentidos distintos. A adesão ao tratamento se refere ao cumprimento de tarefas e procedimentos em busca de resultados positivos para o controle da DM, incluindo também o autocuidado. Este, por sua vez, diz respeito aos comportamentos e posições adotadas pelo indivíduo no cotidiano de convívio com a doença crônica que podem tanto acarretar benefícios quanto agravos à sua saúde (Greco-Soares \& Dell'Aglio, 2017). Estudo desenvolvido com 122 adolescentes com diagnóstico de DM1, em atendimento em um serviço do Sistema Único de Saúde (SUS) mostrou que quanto melhor autocuidado maior a adesão, e quanto maior adesão e autocuidado, menor os valores de hemoglobina glicada (Greco-Soares \& Dell'Aglio, 2017).

Ainda, durante as entrevistas observou-se dificuldade de alguns adolescentes em realizar as atividades de autocuidado em detrimento do estresse causado pelo convívio com a doença crônica e sentimentos como ansiedade.

"[...] quem faz a insulina é minha mãe, mas pelas poucas vezes que eu fiz fiquei um pouco nervoso porque era a primeira vez." (A8, 13 anos)

"Eu fico nervosa, eu tenho crise de ansiedade e quando eu vou fazer eu começo a tremer por causa do nervosismo." (A8, 13 anos)

"Eu não sei... eu tenho muito medo de comer pouco e passar mal depois. Dai eu costumo comer muito em uma hora e depois de duas horas eu como de novo, o que acaba dando alta (insulina)." (A8, 13 anos)

A adolescência é permeada por diversas transformações hormonais, descoberta e formação do caráter e personalidade e quando acrescidas a necessidade de alterações nos hábitos de vida pode gerar um conflito interno no adolescente, dificultando a aceitação da doença e o tratamento. Portanto, essas dificuldades limitam o adolescente no desenvolvimento do autocuidado e a responsabilidade dos cuidados com os níveis glicêmicos recai, geralmente, sobre os familiares (Andrade, 2014). Acerca disso, a literatura aponta que os familiares também sofrem diante do diagnóstico de DM, e expressam principalmente sentimentos de culpa e medo. Além disso, a maioria dos familiares não possui conhecimento acerca da DM e sentem-se limitados para auxiliar o adolescente na rotina de cuidados (Gomes, Moreira, Silva, Motta, \& Soo, 2019).

Por conseguinte, diante das limitações dos adolescentes e suas famílias torna-se imprescindível a atuação dos profissionais de saúde, em especial do enfermeiro, na atenção 
à saúde dessa população. O enfermeiro possui potencial para suprir as lacunas existentes no cotidiano de cuidado dos adolescentes que vivem com DM e suas famílias, visto que eles os acompanham com maior frequência e, portanto, são capazes de identificar suas reais necessidades. Desse modo, o enfermeiro pode planejar ações de cuidado embasadas nas dúvidas e sentimentos desses indivíduos, auxiliando tanto na adesão ao tratamento e promoção autocuidado quanto na redução de sintomas psicológicos nos adolescentes e suas famílias (Gomes et al., 2019).

Ademais, no discurso dos adolescentes a DM trouxe alterações significativas e difíceis para suas vidas, principalmente relacionadas às alterações nos hábitos alimentares.

\section{"[...] Foi difícil acostumar porque era tudo integral, tudo diferente, daí era difícil." (A8, 13 anos)}

"Antes de eu descobrir que tinha diabetes eu comia de tudo, só que dai depois quando minha madrasta me encaminhou para o médico de lá me falaram que não podia comer muito arroz, não podia comer doce. Bem difícil. Ver todo mundo comer e não poder comer..." (A7, 14 anos)

Porém outros adolescentes relatam conseguirem conviver normalmente com a doença, apontando-a como algo positivo.

"Não mudou muito, pra mim ficou quase a mesma coisa. A única coisa que mudou foi meu café e os lanches, antigamente eu não era obrigado a fazer lanche, hoje em dia eu tenho que fazer, se não eu passo mal, obrigatório. Muitas vezes tá alta, é a pior parte, a glicose alta." (A3, 13 anos)

"Tipo muita gente reclama da diabetes, que deixa de fazer muita coisa, comer doce, mas eu não... eu acho que sei lá, vem mais pra ajudar, por incrível que pareça. A gente passa a ter mais responsabilidade com a gente mesmo. Ter um cuidado pro futuro já. Muita gente nessa idade não se cuida tanto com o corpo. Já o diabético, ele se cuida mais." (A4, 17 anos)

Os enunciados dos adolescentes apontam que a maior dificuldade encontra-se em alterar os hábitos alimentares, corroborando com estudo realizado com crianças e adolescentes acompanhados por ambulatório de hospital universitário, os quais apontaram a alimentação saudável como principal empecilho no tratamento da DM (Bertin et al., 2016).

O sentimento de aceitação e positividade com relação à doença, mencionado nas falas, pode estar relacionado com a capacidade de resiliência do indivíduo. Para Castro e MorenoJiménez (2007) a resiliência, relacionada às condições crônicas, se refere a capacidade de adaptação, de progressão positiva e de superação da doença. Além disso, a resiliência tem relação também com os fatores de proteção que envolvem o indivíduo e o auxiliam nos desafios de conviver com a doença crônica. Dentre esses fatores destacam-se 
características pessoais, vínculo com adultos de referência; e rede de apoio familiar, grupos de pares e serviços de saúde (Cassarino-Perez \& Dell'Aglio, 2015).

As alterações na alimentação também foram elencadas como negativas para a interação social dos adolescentes, prejudicando relações comuns na adolescência.

"O único problema é a bebida né, agora eu to ficando jovem e eu não posso beber né!? Cerveja... vodka... essas coisas. Daí normalmente eu me excluo dessas coisas também." (A9, 14 anos)

"Quando eu vou sair com meus amigos, se eles compram um refri ou alguma coisa assim, eu fico meio de fora porque eu não posso tomar, então é um negócio que meio que dá uma excluída ali né!?” (A10, 16 anos)

Durante a adolescência a necessidade de sentir-se incluído em grupos de pares é maior, a fim de o adolescente sentir-se aceito pela sociedade. Porém, os grupos de pares tendem a aumentar a suscetibilidade aos comportamentos de risco como ingestão de bebidas alcoólicas e o tabaco, potencializando riscos para a estabilidade glicêmica e futuras complicações da DM (Gaete, 2015).

Com relação à prática de exercícios físicos, os adolescentes entrevistados relataram não praticarem atividades físicas por não gostarem ou não julgarem necessário.

"Ultimamente não tenho feito (exercícios), tenho me baseado mais pela insulina, porque a insulina que eu faço, a ultrarrápida dá um pouco mais de liberdade sabe, dá pra comer o que quiser fazendo a contagem dos carboidratos certinho." (A4, 17 anos)

"Eu fazia academia, agora eu parei. Não gostei muito." (A2, 13 anos)

Já os adolescentes que relataram aderir à prática de exercícios físicos, dizem fazê-lo nas aulas de educação física que realizam na escola.

“Eu pratico na escola, na educação física.” (A5, 14 anos)

"Eu faço exercícios duas vezes por semana, na escola." (A8, 13 anos)

Acerca da prática de exercícios físicos, estudo desenvolvido com 51 adolescentes com DM1, 49\% não praticavam exercício físico regular, 20\% praticavam um a dois períodos por semana $31 \%$ três ou mais períodos por semana (Pereira, Neto, Moleiro, \& Gama, 2015). A prática de exercícios físicos além de prevenir o surgimento da doença, auxilia na redução das taxas de glicose no sangue, na ação da insulina na circulação sanguínea, diminui peso e riscos de doenças cardiovasculares, melhora as capacidades cardiorrespiratórias, reduz a pressão arterial, controla a hemoglobina glicada e melhora a sensibilidade à insulina. 
Portanto, a combinação entre a prática regular de exercícios físicos e uma dieta equilibrada se caracteriza como estratégia fundamental para o controle glicêmico, havendo necessidade de intervenção profissional, a fim de conscientizar os adolescentes acerca da importância do exercício físico no controle da DM (Marçal, Alexandrino, Cortez, \& Bennemann, 2018).

\section{CONSIDERAÇÕES FINAIS}

A partir do desenvolvimento desta invetigação qualitativa, por meio das entrevistas, foi possível conhecer como os adolescentes que vivem com diabetes mellitus desenvolvem o autocuidado. Nessa direção, destaca-se a importância da pesquisa qualitativa para obtenção dos resultados, pois ela permite um aprofundamento do fenômeno estudo, considerando características, subjetividades e contextos. Com isso, pôde-se compreender a percepção que os adolescentes têm acerca do autocuidado e identificar as dificuldades encontradas por eles no convívio com a doença crônica, evidenciando lacunas no cuidado.

Portanto, conclui-se que os adolescentes entendem o autocuidado como as atividades cotidianas que precisam e realizam em razão do convívio com a DM. Esses adolescentes possuem autonomia na realização do autocuidado e conhecimento sobre a doença. Além disso, as principais limitações para o autocuidado se referem à adoção de hábitos alimentares saudáveis e a prática regular de exercícios físicos.

Esses achados reforçam importantes aliados para o planejamento das ações de cuidado direcionadas aos adolescentes que vivem com DM e suas famílias, ao passo que apontam aos profissionais de saúde as fragilidades enfrentadas no manejo da doença que podem culminar em complicações agudas ou crônicas e internações hospitalares. Logo, compete também aos profissionais de saúde orientar e englobar a família no tratamento da DM, uma vez que ela exerce importante influência na adesão do adolescente ao tratamento em razão do apoio que oferecem.

Com relação às contribuições desta pesquisapara a prática sugere-se que os profissionais de saúde acompanhem e estimulem o adolescente na realização do autocuidado, dotando-o de conhecimento a fim de torná-lo independente e responsável, capaz de decidir sobre sua saúde de maneira consciente e assistida. Como limitação do estudo aponta-se o delineamento transversal, que realiza um recorte da população em determinado período de tempo, visto que para melhor conhecimento acerca do autocuidado de adolescentes que vivem com DM seria interessante realizar um acompanhamento prolongado, a fim de 
compreender de maneira aprofundada o problema em questão. Assim, sugere-se a realização de estudo etnográfico com essa população, que busque compreender também os fatores sócio-culturais relacionados ao autocuidado.

\section{REFERÊNCIAS}

Andrade, M. L. M. S. (2014). A saúde e os estilos de vida dos jovens adultos com diabetes tipo 1. (Tese de Doutorado em Educação, Universidade de Lisboa, Portugal). Disponível em https://core.ac.uk/download/pdf/61473432.pdf

Bertin, R. L., Elizio, N.P.S., Moraes, R.N.T., Medeiros, C.O., Fiori, L.S., \& Ulbrich, A.Z. (2016). Perepções do cotiano alimentar de crianças e adolescentes com diabetes mellitus tipo 1. Revista Contexto \& Saúde, 16 (30), 100-109. doi: 10.5020/18061230.2016.p371

Bertonhi, L. G., e Dias, J. C. R. (2018). Diabetes mellitus tipo 2: aspectos clínicos, tratamento e conduta dietoterápica. Revista de Ciências Nutricionais On, 2(2), 1-10. Disponível em http://unifafibe.com.br/revistasonline/arquivos/cienciasnutricionaisonline/sumario/62/18042018212025.pdf

Brasil. Ministério da Saúde. (2015). Estatuto da Criança e do Adolescente. (13 ed). Brasília, DF: Ministério da Saúde.

Braun, V., \& Clarke, V. (2006, december 1). Using thematic analysis in psychology. Qualitative Research in Psychology, 77-101. doi: 10.1191/1478088706qp063oa

Cassarino-Perez, L., \& Dell'Aglio, D. D. (2015) Processos de resiliência em adolescentes com diabetes melittus tipo I. Psicologia em Estudo, 20(1), 45-56. doi: 10.4025/psicolestud.v20i1.24035

Castro, E. K., \& Moreno-Jiménez, B. (2007). Resiliencia em niños enfermos crônicos: Aspectos teoricos. Psicologia em Estudo, 12(1), 81-86. doi: 10.1590/S1413-73722007000100010

Cavini, F. L., Gonçalves, K. A., Samara, M. C., Moreira, D. S., \& Rescks, Z. M. R. (2016). Vivências de adolescentes com diabetes: Uma abordagem fenomenológica. Revista de Enfermagem UFPE online, 10(2), 805-13. doi: 10.5205/reuol.6884-59404-2-SM-1.1002sup201615

Conte, A. F. \& JeneraL, R. B. R. (2018). Sentimentos vivenciados pelos adolescentes portadores de diabetes mellitus tipo 1. Revista da Faculdade de Ciências Médicas de Sorocaba, 20(4), 218-22. doi: 10.23925/1984-4840.2018v20i4a7

Costa, M., e Gameiro, M. G. H. (2016). Autocuidado dos adolescentes com diabetes mellitus tipo 1: responsabilidade no controlo da doença. Revista de Enfermagem Referência, (9), 9-19. doi: 10.12707/RIV16010

Espírito-Santo, M. B., Souza, L. M. E., Souza, A. C. G, Ferreira, F. M., e Silva, C. N. M. R. (2012). Adesão dos portadores de diabetes mellitus ao tratamento farmacológico e não farmacológico na atenção primária à saúde. Revista Enfermagem, 15(1), 88-101. Disponível em http://periodicos.pucminas.br/index.php/enfermagemrevista/article/view/3275

Ferreira, L. E., Zanatta, E. A., Brum, M. L. B., Nothaft, S. C., \& Motta, M. G. C. (2013). Diabetes mellitus sob a ótica do adolescente. Cogitare Enfermagem, 18(1), 71-7. Disponível em https://www.lume.ufrgs.br/bitstream/handle/10183/83609/000895917.pdf?sequence=1

Gaete, V. (2015). Desarrollo psicosocial del adolescente. Revista Chilena de Pediatría, 86 (6). doi: 10.1016/j.rchipe.2015.07.005 
Gomes, G. C., Moreira, M. A. J., Silva, C. D., Mota, M. S., Nobre, C. M. G., \& Rodrigues, E. F. (2019). Vivências do familiar frente ao diagnóstico de diabetes mellitus na criança/adolescente. Journal of Nursing and Health, 9(1). doi: 10.15210/jonah.v9i1.13393

Greco-Soares, J. P., \& Dell'Aglio, D.D. (2016). Relações entre qualidade de vida e diabetes mellitus tipo 1 na adolescência. Contextos Clínicos, 9(2). doi: 10.4013/ctc.2016.92.02

Greco-Soares, J. P., \& Dell'Aglio, D.D. (2017). Treatment adherence in adolescents with type 1 diabetes mellitus. Psicologia, Saúde \& Doenças, 18(2). doi: 10.15309/17psd180204

Marçal, D. F. S., Alexandrino, E. G., Cortez, L. E. R., \& Bennemann, R. M. (2018). Efeitos do exercício físico sobre diabetes mellitus tipo 1: uma revisão sistemática de ensaios clínicos e randomizados. Journal of Physical Education, 29. doi: 10.4025/jphyseduc.v29i1.2917

Minayo, M. C. S. (2014). O desafio do conhecimento: pesquisa qualitativa em saúde (14 ed.). São Paulo: Hucitec.

Moreira, H., \& Caleffe, L. G. (2008). Metodologia da pesquisa para o professor pesquisador. Rio de Janeiro: DP \& A.

Pereira, E., Neto, S., Moleiro, P., \& Gama, E. (2015). Exercício Físico em Adolescentes com Diabetes Mellitus Tipo 1. Acta Pediátrica Portuguesa, 46, 311-7. Disponível em https://www.researchgate.net/publication/283297302_Physical_Exercise_in_Adolescents_with_Type_1_Dia betes_Exercicio_Fisico_em_Adolescentes_com_Diabetes_Mellitus_Tipo_1

Souza, A. B. L., \& Cruz, A. C. D. (2015, Março). Implantação da caderneta do adolescente: Relato do município de Manaus. Adolescência \& Saúde, 12(1), 52-59. Disponível em http://www.adolescenciaesaude.com/detalhe_artigo.asp?id=491

Viero, V. S. F., Farias, J. M., Ferraz, F., Simões, P. W., Martins, J. A., \& Ceretta, L. B. (2015). Educação em saúde com adolescentes: análise da aquisição de conhecimentos sobre temas de saúde. Escola Anna Nery, 19(3). doi: 10.5935/1414-8145.20150064 